\title{
Apoptotic Cell Death in Mammalian Cells
}

\author{
Sahar M Gerbil,Hoda M Elsayed, Eman E. Abu-Dief \\ Department of Histology, Faculty of Medicine, Sohag University, Egypt
}

\section{Review article}

\section{Abstract}

Cell death is a highly organized pathophysiological process, fundamental for normal life and development. Balance between cell proliferation and cell death is essential for the development and maintenance of multicellular organisms. Despite the different causing stimuli, cell death mode typically follows one of two patterns. The first is programmed cell death (PCD) while the second pattern is necrosis. PCD include more than 15 types, the most common types are three include; apoptosis, autophagic cell death and programmed necrosis (necroptosis). Disorders of either process have pathologic consequences and can lead to disturbed normal embryogenesis, neurodegenerative diseases, or cancer development. This review article discusses the apoptotic cell death types, molecular pathwayandmorphological changes.

\section{Introduction}

Apoptosisas a PCD type induces biochemical events that orchestrate embryogenesis, body homeostasis, immune system, and cancer.It is characterized by activation of specific proteins called caspases which mediates cleavage of vital cellular proteins and chromatin condensation. The expression 'apoptosis' describes a specificmorphology of cell death. Apoptosis disturbance disrupts the normal equilibrium between cell survival and cell death causing cancer development (Setia and Sanyal, 2012).

Apoptosis could be induced by different stimuli including; irreparable damage of the DNA, disturbance of the cell cycle,cell metabolism aberrations, depletion of extracellular molecules that promote cell or inhibit cell death, death receptors activating ligands, growth factors withdrawal, calcium influx, free radicals, viral infections and bacterial toxins, radiation and chemotherapy drugs(Ghatage et al., 2012).
Apoptosis could be inhibited byinhibitor of apoptosis proteins (IAP) that function as inhibitors of endogenous caspase, participant in cell cycle regulation and in receptormediated signal transductionmodulation. Survivinis structurally a unique IAP that acts through inhibition of caspase- 9 activation and byenhancing microtubule stability in metaphase spindle formation during mitosis(Liston et al., 2003).

Apoptosis can be classified as caspase dependent and caspase independent apoptosis

\section{Caspaseindependent apoptosis}

Apoptosis is induced by, various factors, like; apoptosisinducing factor (AIF), endonuclease G, Omi/HtrA2. AIFis a mitochondrial protein that induces PCD. AIF is activated as cell killer when it is released to the cytosol, then trans located to the nucleus and triggers, together with endonuclease $\mathrm{G}$, peripheral chromatin condensation, and DNA loss. AIF release is 
independent of the caspase cascade but triggered by the lysosomal protease cathepsin D. However the anti-apoptotic protein heat shock protein 70 could interacts with AIF and prevents its apoptogenic effects(Bröker et al., 2005).

\section{Caspase dependent apoptosis}

Caspases are aspartate-specific cysteine endoproteases that represent critical links in cell networks controlling apoptosis. They have been classified by their known roles in apoptosis (caspase-3, -6,-7, -8, and 9 ), and in inflammation (caspase-1, -4, -5, 12). They include both initiator and executioner caspases. The initiator caspases8 and -9 normally exist as inactive forms that are activated by dimerization and not by cleavage. The executioner caspases-3, -6, and -7 are produced as inactive procaspase dimers that must be cleaved by initiator caspases to be active(Hussein, 2005).

There are two different subtypes of caspase dependent apoptosis that morphologically similar, but can be triggered through different biochemical routes. The two alternative pathways of apoptosis are; an extrinsic one, a death receptor-initiated caspase 8 dependent pathway and an intrinsic one, a mitochondrion-initiated caspase 9 (Hekimi et al., 2016) and ER stress initiated caspase 4 (12 in rodents) dependent pathway(Banjerdpongchai et al., 2010).

The extrinsicapoptotic pathway is mediated by activation of cell death receptors on the cell surface by specific ligand forming cell death domains. These death domains attract the intracellular adaptor protein Fasassociated death domain (FADD) that recruits members of inactive caspase proteases (caspase-8, caspase-2) and form death-inducing signalling complex (DISC). Cleavage of the latter activate the initiator caspase-8 that amplifies the apoptotic cascade by activation of executioner caspases (caspase-3, -7, and -6 )(Liu et al., 2017).

The intrinsic apoptotic pathway is either induced by mitochondrial pathway, regulated by B-cell lymphoma 2 (Bcl-2) family that contains 25 known proteins. These proteins are either apoptosis stimulatory (pro-apoptotic) or inhibitory (anti-apoptotic) with a delicate balance in between. Apoptotic stimuli as DNA damage stimulate members of the Bcl-2 family that activate mitochondrial membrane proapoptotic proteins as (Bax or Bak), or inhibit anti-apoptotic proteins. Activated proapoptotic proteins bind to mitochondrial outer membrane causing its permeability and release of essential pro-apoptotic factors and cytochrome $c$. In the cytoplasm, cytochrome $c$ together with the apoptotic factors form "apoptosome" complex, which activates initiator caspase- 9 that activate executioner caspases, (caspase-3, -7, and -6 ) the same end as extrinsic pathway (Morrill and $\mathrm{He}, 2017$ ).

ER normally regulates protein synthesis and intracellular calcium homeostasis. ER stress caused by accumulation of unfolded proteins triggers apoptosis either through caspase 12 oligomerization and cleavage or release of calcium. The latter leads to the activation of several transcription factors that induce cytochrome $c$ release from the mitochondria and apoptosome formation. Caspase 12 acts as a direct activator of caspase 9, independently of mitochondrial cytochrome $c$ release and apoptosome formation(Di Sano et al., 2006).

Caspase- 3 is the most important of all caspases, it can cause DNA and chromatin damage, intracellular transport disruption, cytoskeleton re-arrangement, and disrupt 
signal transduction and cell division. Once execution caspases are activated, theycannot be stopped and cell death is certain. Production of cellular fragments at the final stage of apoptosis are quickly recognized and engulfed and digested by the macrophages or the surrounding neighbouring cells(Morrill and He, 2017).

\section{Apoptosis morphological changes}

Apoptosis is characterized by three cellular morphological stages (figure 1); an initial one in which early cellular morphological changes like rounding-up of the cell, pseudopods retraction, cell volume reduction causing cell shrinkage. The second stage shows membrane blebbing (loss of membrane lipid asymmetrybut maintenance of its integrity until the final stages of the process)and plasma membrane formation of cell membrane vesicles containing different cellular components that bud off from the main body of the cell (apoptotic bodies). The final stage is characterized by degeneration of nuclear material and pyknosis (chromatin condensation), nuclear fragmentation (karyorrhexis), classically with little or no ultrastructural modifications of cytoplasmic organelles. Cellular fragmentations and apoptotic bodiesthen are engulfed by resident phagocytes without inducing inflammation unlike necrosis where cell damage and lyses occur releasing cell contents and inducing inflammation(Porter et al., 2000; Peixoto et al., 2017).

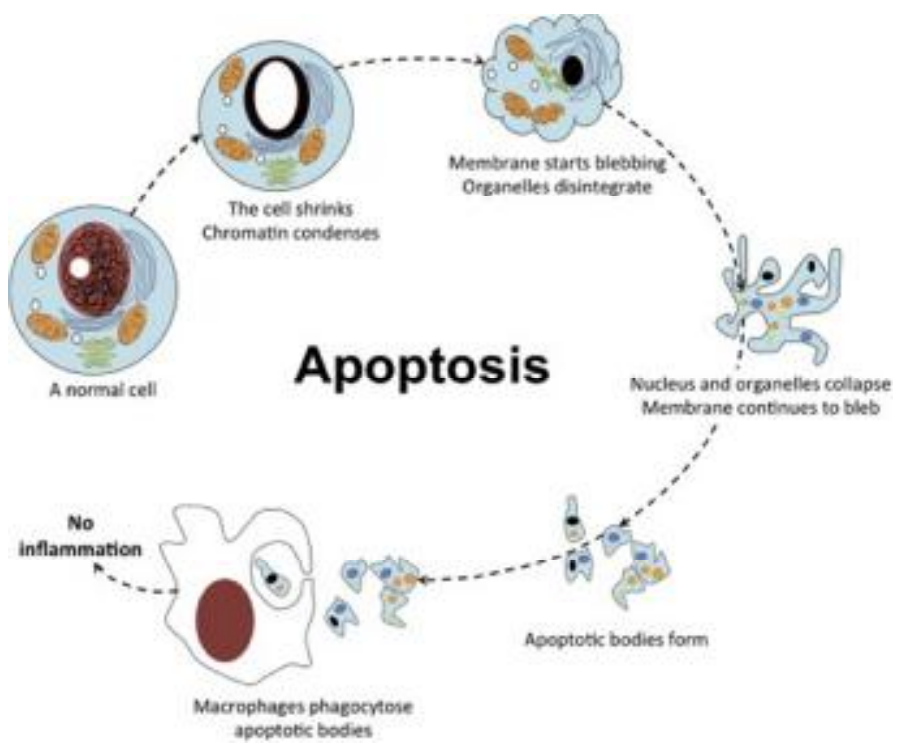

Figure 1:diagram of apoptotic cell death morphological changes (Abou-Ghali and Stiban, 2015)

\section{Conclusion}

Apoptosis is an important biological mechanism involved in normal development and its disturbance is involved in many diseases. The elucidations of the molecular mechanisms and the morphology of

\section{References}

apoptosis have great aimpotence in studying the underlying molecular mechanisms of different diseases for putting therapeutic strategies. 
1- Abou-Ghali M and Stiban J (2015) Regulation of ceramide channel formation and disassembly: Insights on the initiation of apoptosis. Saudi Journal of Biological Sciences 22:760-772.

2- Banjerdpongchai R, Kongtawelert $P$, Khantamat O, Srisomsap C, Chokchaichamnankit D, Subhasitanont P and Svasti J (2010) Mitochondrial and endoplasmic reticulum stress pathways cooperate in zearalenone-induced apoptosis of human leukemic cells. Journal of Hematology \& Oncology3:50-50.

3- Bröker LE, Kruyt FAE and Giaccone G (2005) Cell Death Independent of Caspases: A Review. Clinical Cancer Research11:3155.

4- Di Sano F, Ferraro E, Tufi R, Achsel T, Piacentini $M$ and Cecconi F (2006) Endoplasmic reticulum stress induces apoptosis by an apoptosome-dependent but caspase 12-independent mechanism. J Biol Chem 281:2693-2700.

5- Ghatage D, Gosavi S, Ganvir $S$ and Hazarey V (2012) Apoptosis: Molecular mechanism. Journal of Orofacial Sciences4:103-107.

6- Hekimi S, Wang Y and Noë A (2016) Mitochondrial ROS and the Effectors of the Intrinsic Apoptotic Pathway in Aging Cells: The Discerning Killers! Frontiers in Genetics7.
7- Hussein MR (2005) Apoptosis in the ovary: molecular mechanisms. Human reproduction update11:162-177.

8- Liston P, Fong WG and Korneluk RG (2003) The inhibitors of apoptosis: there is more to life than $\mathrm{Bcl} 2$. Oncogene22:8568.

9- Liu B, Sun R, Luo H, Liu X, Jiang M, Yuan C, Yang L and Hu J (2017) Both intrinsic and extrinsic apoptotic pathways are involved in Toll-like receptor 4 (TLR4)-induced cell death in monocytic THP-1 cells. Immunobiology222:198-205.

10- Morrill S and He DZZ (2017) Apoptosis in inner ear sensory hair cells. Journal of Otology 12:151-164.

11- Peixoto MS, de Oliveira Galvão MF and Batistuzzo de Medeiros SR (2017) Cell death pathways of particulate matter toxicity. Chemosphere 188:32-48.

12- Porter SN, Howarth GS and Butler RN (2000) Non-steroidal anti-inflammatory drugs and apoptosis in the gastrointestinal tract: potential role of the pentose phosphate pathways. European Journal of Pharmacology397:1-9.

13- Setia S and Sanyal SN (2012) Upregulation of intrinsic apoptotic pathway in NSAIDs mediated chemoprevention of experimental lung carcinogenesis. Pharmacological Reports64:615-624. 